\title{
The Effect of Prenatal Cadmium Exposure on Attention-deficit/Hyperactivity Disorder in 6-Year-old Children in Korea
}

\author{
Woosung Kim', Yoonyoung Jang ${ }^{1}$, Youn-Hee Lim², Bung-Nyun Kim³ , Choong Ho Shin' ${ }^{4}$, Young Ah Lee ${ }^{4}$, \\ Johanna Inhyang Kim ${ }^{3}$, Yun-Chul Hong ${ }^{1,2,5}$
}

${ }^{1}$ Department of Preventive Medicine, Seoul National University College of Medicine, Seoul, Korea; ${ }^{2}$ Institute of Environmental Medicine, Seoul National University Medical Research Center, Seoul, Korea; ${ }^{3}$ Division of Children and Adolescent Psychiatry, Department of Psychiatry, Seoul National University Hospital, Seoul, Korea; ${ }^{4}$ Department of Pediatrics, Seoul National University Children's Hospital, Seoul, Korea; ${ }^{5}$ Environmental Health Center, Seoul National University College of Medicine, Seoul, Korea

Objectives: Prenatal cadmium (Cd) exposure may be associated with attention-deficit/hyperactivity disorder (ADHD) in children. Therefore, the objective of this study was to examine the relationship between $\mathrm{Cd}$ exposure during gestation and ADHD at 6 years of age. Methods: As part of an ongoing cohort study (the Environment and Development of Children study), 479 mother-child pairs from Seoul, Korea were included for analysis between 2008 and 2011. The whole blood concentration of $\mathrm{Cd}$ was analyzed using atomic absorption spectrophotometry. The parents were surveyed about ADHD behaviors in their children at age 6. Multivariable linear regression models were used to investigate the relationship between prenatal exposure to $C d$ and ADHD at 6 years of age.

Results: Increased prenatal Cd concentrations were associated with increased scores for ADHD for girls, but not for boys, at age 6. A 2 -fold increase in the prenatal Cd level was significantly associated with a $22.3 \%$ (95\% confidence interval, 11.6 to 34.1 ) increase in ADHD in girls at 6 years of age, as indicated by the linear regression model.

Conclusions: Our results identified significant associations between prenatal $\mathrm{Cd}$ exposure and ADHD scores in 6-year-old girls.

Key words: Cadmium, Preschool child, Attention-deficit/hyperactivity disorder, Republic of Korea

\section{INTRODUCTION}

Cadmium (Cd), a heavy metal, is dispersed throughout the earth's soil both naturally and from human activities such as the burning of fossil fuels, the incineration of waste, industrial

Received: June 29, 2019 Accepted: November 6, 2019

Corresponding author: Yun-Chul Hong, MD, PhD

Department of Preventive Medicine, Seoul National University College of Medicine, 103 Daehak-ro, Jongno-gu, Seoul 03080, Korea

E-mail: ychong1@snu.ac.kr

This is an Open Access article distributed under the terms of the Creative Commons Attribution Non-Commercial License (https://creativecommons.org/licenses/by$\mathrm{nc} / 4.0 /$ ) which permits unrestricted non-commercial use, distribution, and reproduction in any medium, provided the original work is properly cited. smelting procedures, and the use of phosphate-containing fertilizers [1]. Among smokers, tobacco use is the dominant source of $\mathrm{Cd}$ exposure; however, for the general non-smoking population, diet (specifically, the consumption of leafy vegetables, root crops, grains, organ meats, and seafood) is the primary source of exposure $[1,2]$. Cd is known to cause nephrotoxicity [3], and growing evidence also suggests that exposure to $\mathrm{Cd}$ may have adverse neurodevelopmental consequences. Animal studies have described the effects of $\mathrm{Cd}$ on electrophysiological parameters of the brain, changes in neurotransmitter function, and adverse neurobehavioral outcomes [4-8]. In children, studies have reported associations between increased $\mathrm{Cd}$ levels and outcomes such as mental retardation 
$[9,10]$, lower verbal intelligence quotient [11], learning disabilities [12-14], poor reading performance [15], and behavioral problems [16]. However, other human studies have shown contradictory results, failing to detect significant associations between exposure to $\mathrm{Cd}$ and neurodevelopmental outcomes [17-19].

Evidence suggests that the prevalence of attention-deficit/ hyperactivity disorder (ADHD) is greater in males than in females [20]. In the Attention-Deficit Hyperactivity Disorder Observational Research in Europe study, which was a large 24month longitudinal observational study of children aged 6-18 years that was conducted in 10 European countries, the sex distribution of the study subjects varied by country, ranging from a 1:3 to a 1:16 ratio of girls to boys [21]. With regard to individuals with ADHD, such a disparity not only exists in childhood, but also persists into adulthood, as ADHD is more commonly diagnosed in adult males than adult females. A worldwide meta-regression analysis of 11 studies of adults with ADHD found that although the ratio of males to females with ADHD decreased with increasing age, a sex-based difference was still present in adults aged 19 years and over [22]. A pooled analysis of studies of ADHD outcomes estimated the rate of persistence of ADHD into adulthood as approximately $15 \%$ when limited to those who meet the full criteria for ADHD. However, when cases consistent with the Diagnostic and Statistical Manual of Mental Disorders, 4th edition (DSM-IV) definition of $A D H D$ in partial remission were included, the persistence into adulthood was estimated to be as high as about $60 \%$ [23]. Two studies ( 1 in males and 1 in females) on the persistence of ADHD reported that 11 years after the initial diagnosis, $35.0 \%$ of males and $33.3 \%$ of females continued to meet the DSM-IV criteria for $A D H D$, indicating that the persistence of ADHD is similar between the sexes [24]. Despite the apparent sex-based differences, research has mainly focused on ADHD in boys, and studies of ADHD in girls are still limited [25].

In the present study, we used an ongoing cohort of 6-yearold children to examine ADHD symptoms associated with prenatal exposure to $\mathrm{Cd}$, using a linear regression model. In addition, we examined sex-specific effects on ADHD.

\section{METHODS}

\section{Population}

The present study was based on the Environment and Development of Children (EDC) study, a prospective cohort study.
The participants chosen for the EDC study were mothers and children who previously participated in the Congenital Anomaly Study (CAS), another cohort study of birth outcomes. In the CAS cohort study, pregnant women from the metropolitan areas of Seoul and Incheon, Korea, who received prenatal care at local hospitals were recruited. The total number of enrolled pregnant females was 13484 , and subjects were recruited during the second trimester of pregnancy. Of these females, 11085 remained in the study and were followed up until childbirth, between August 2008 and July 2011. Blood and urine samples were collected at the time of enrollment, after subjects had fasted for more than 8 hours. A questionnaire about participants' demographics and lifestyles was administered by trained nurses. In the CAS cohort, 115 children were found to have congenital anomalies. These children, as well as those with invalid addresses $(n=218)$, were excluded from recruitment, and a total of 10752 mothers were eligible for the EDC birth cohort. A sample size of 610 (effect size, $0.017 ; \alpha, 0.05$; power, 0.90 ) was calculated to be sufficient to examine the association between $\mathrm{Cd}$ exposure and other characteristics of the children. The sample size was then inflated by $5 \%$, to 645 , to allow for dropouts. Between 2012 and 2015, we contacted 2085 mothers chosen at random from the 10752 EDC participants, until 726 mother-child pairs were enrolled in the present study (response rate, $31 \%$ ). We conducted follow-up assessments when the children were approximately 6 years of age, between March 2015 and December 2017. The children underwent health examinations at the Seoul National University Hospital in Jongno-gu, Seoul, Korea. The mothers' depressive symptoms and the children's dietary habits were assessed using the Center for Epidemiologic Studies Depression Scale and food frequency questionnaires, respectively.

Neurobehavioral tests for ADHD were administered to the 6-year-old children between 2015 and 2017. The Korean version of the ADHD rating scale (K-ARS) was used to measure ADHD symptoms. At the follow-up examination, urine and blood samples were collected, and physical examinations were conducted, after the children had fasted for more than 8 hours. For prenatal measurements, we assessed blood lead $(\mathrm{Pb})$ and $\mathrm{Cd}$ concentrations in whole blood samples during the second trimester of pregnancy, between 14 weeks and 27 weeks (mean, 20 weeks) of gestation. Whole blood heavy metal levels were quantified using atomic absorption spectrophotometry.

After excluding those who did not complete the K-ARS assessment $(n=77)$, those without maternal $(n=63)$ or child 
$(\mathrm{n}=22)$ blood $\mathrm{Cd}$ measurements, and those with missing covariate values $(n=2), 479$ mother-child pairs were included in the present analyses.

\section{Assessments of Attention-deficit/Hyperactivity Symptoms}

The parents completed a questionnaire about demographic data and related factors, including socioeconomic status, secondhand smoking exposure, parental age, parental education level, and medical and obstetric histories of themselves and their children. Parental education level was classified as high school graduate or less ( $\leq 12$ years of education) or university or above (more education than a high school graduate). We used a previously validated translated version of the ADHD rating scale (ARS) called the K-ARS to assess ADHD symptoms. The ARS, which was developed to assess the severity of ADHD symptoms [26], consists of 18 items that reflect the DSM-IV diagnostic criteria for ADHD. Since the K-ARS is a translated version of the ARS and therefore contains identical content to the ARS, it was selected as an adequate method for use on Korean subjects. Each item of the K-ARS is rated on a 4-point Likert scale (0-3 points, total score 0-54), with potential responses of "never or rarely," "sometimes," "often," or "very often."

\section{Measurements of Blood Lead and Cadmium}

Whole blood was collected from the subjects in trace element ethylenediaminetetraacetic acid tubes, and the samples were analyzed using atomic absorption spectrophotometry ( $240 Z$ AA Zeeman spectrometer, Agilent, Santa Clara, CA, USA), using the Environmental Calibration Standard (Part \#5183-4688, Agilent), with ammonium phosphate monobasic $\left(20 \% \mathrm{NH}_{4} \mathrm{H}_{2} \mathrm{PO}_{4}\right.$, Sigma-Aldrich, St. Louis, MO, USA) and Triton X-100 (Sigma-Aldrich) as reagents. Once collected, the samples were frozen and stored at $-20^{\circ} \mathrm{C}$. Before analysis, the blood samples were brought to room temperature and pretreated using a Vortex Genie 2 mixer (Scientific Industries, Bohemia, NY, USA) and an ES 220A balance (Precisa Gravimetrics AG, Dietikon, Switzerland). The samples were diluted with a matrix modifier reagent (Triton X-100 and ammonium hydrogen phosphate monobasic), then assayed using the matrix-matched calibration curve method (the standard addition method). The method detection limit (MDL) was calculated according to the following formula:

$$
\mathrm{MDL}=t_{(n-1,1-\alpha=0.99)} \times S
$$

Table 1. Characteristics of participants $(n=479)$

\begin{tabular}{lrrr}
\hline Characteristics & Overall & Boys & Girls \\
\hline Total & $479(100)$ & $256(53.4)$ & $223(46.6)$ \\
Age $(\mathrm{mo})$ & $71.1 \pm 1.6$ & $71.1 \pm 1.6$ & $71.1 \pm 1.6$ \\
Body mass index $\left(\mathrm{kg} / \mathrm{m}^{2}\right)$ & $15.8 \pm 1.7$ & $15.8 \pm 1.7$ & $15.8 \pm 1.7$ \\
Maternal age (y) & $31.4 \pm 3.6$ & $31.5 \pm 3.6$ & $31.2 \pm 3.5$ \\
Maternal blood cadmium level $(\mu \mathrm{g} / \mathrm{L})$ & $0.7 \pm 0.3$ & $0.7 \pm 0.3$ & $0.7 \pm 0.3$ \\
Maternal blood lead level $(\mu \mathrm{g} / \mathrm{dL})$ & $1.4 \pm 0.4$ & $1.4 \pm 0.5$ & $1.3 \pm 0.3$ \\
Maternal education level & & & \\
High school or less & $75(15.6)$ & $40(15.6)$ & $35(15.7)$ \\
$\quad$ University or above & $404(84.3)$ & $216(84.4)$ & $188(84.3)$ \\
Current smoker & $31(6.4)$ & $19(7.4)$ & $12(5.4)$ \\
Secondhand smoke exposure & & & \\
$\quad$ No & $87(18.2)$ & $44(17.2)$ & $43(19.3)$ \\
Yes & $392(81.8)$ & $212(82.8)$ & $180(80.7)$ \\
Parity & & & \\
First child & $292(61.0)$ & $156(60.9)$ & $136(61.0)$ \\
Second child or above & $187(39.0)$ & $100(39.0)$ & $87(39.0)$ \\
K-ARS scores & & & \\
Total & $6.2 \pm 5.8$ & $6.9 \pm 5.7$ & $5.4 \pm 5.9$ \\
Attention deficit & $3.4 \pm 3.2$ & $3.9 \pm 3.1$ & $3.0 \pm 3.3$ \\
Hyperactivity & $2.7 \pm 3.1$ & $3.0 \pm 3.0$ & $2.3 \pm 3.0$ \\
\hline
\end{tabular}

Values are presented as mean \pm standard deviation or number (\%).

SD, standard deviation; K-ARS, Korean attention-deficit/hyperactivity disorder rating scale.

where:

$\mathrm{MDL}=$ method detection limit

$t_{(n-1,1-\alpha=0.99)}=$ Student's $t$-value appropriate for a singletailed 99th-percentile $t$ statistic and a standard deviation estimate with $\mathrm{n}-1$ degrees of freedom

$S=$ sample standard deviation of the replicate spiked-sample analyses

Seven samples were used to calculate the MDL, so 3.14 was multiplied by the sample standard deviation (as found in $\mathrm{Ad}$ dendum Table 1 of the Definition and Procedure for the Determination of the Method Detection Limit, Revision 2, United States Environmental Protection Agency). The MDL values for lead and cadmium were found to be $0.091 \mu \mathrm{g} / \mathrm{dL}$ and $0.031 \mu \mathrm{g} / \mathrm{L}$, respectively.

\section{Statistical Analysis}

Blood Cd concentrations were transformed by $\log _{2}$ (base 2) to obtain a normal distribution. In order to investigate the relationship between prenatal $\mathrm{Cd}$ concentrations and ADHD scores at age 6 , we used generalized additive models. In the examination of the regression curve for the generalized addi- 
tive model, we assumed a linear relationship. We constructed a regression model for $\mathrm{Cd}$ exposure and ADHD scores to estimate the overall linear effects of $C d$ exposure on ADHD scores.

We modeled scores on the K-ARS as a Poisson distribution and estimated the effects of $\mathrm{Cd}$ exposure on the total and subcategory scores for ADHD. All models controlled for potential covariates including sex (male or female), parity, birth weight $(\mathrm{kg})$, mother's body mass index (BMl, $\left.\mathrm{kg} / \mathrm{m}^{2}\right)$, mother's education level, mother's age, and mother's smoking status. To examine potential sex-based differences, we stratified our samples by sex. In our sensitivity analysis, we included concurrent child blood $\mathrm{Cd}$ concentration measured at the age of 6 and maternal blood $\mathrm{Pb}$ concentration in our main model.

The statistical analyses were conducted using SAS version 9.4 (SAS Institute, Inc., Cary, NC, USA) and R version 3.2.1 (https:// cran.r-project.org/bin/windows/base/old/3.2.1/). Two-tailed $p$ values of less than 0.05 were considered to indicate statistical significance.

\section{Ethics Statement}

We obtained informed consent from all participants, and the study protocol was approved by the Institutional Review Board of the Seoul National University College of Medicine (IRB No. 1201-010-392).

\section{RESULTS}

The characteristics of the study population regarding maternal and child age, sex, BMI, maternal education level, parity,

K-ARS total score in 6-year-old girls

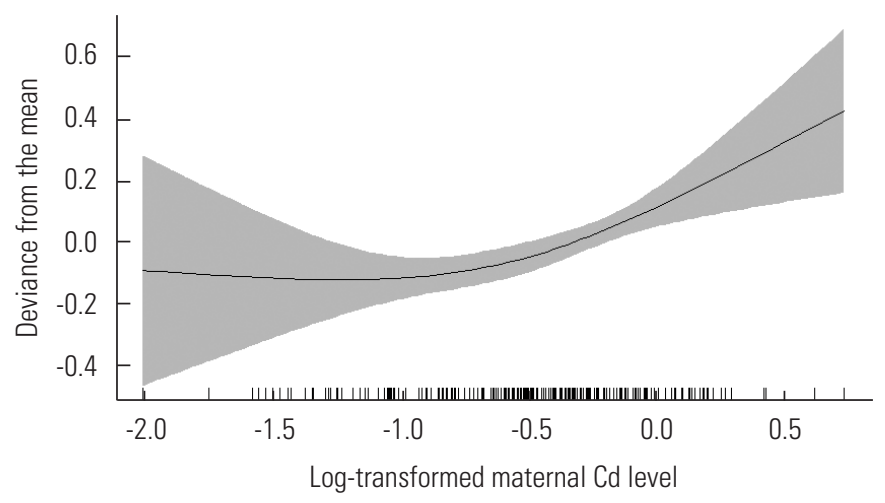

Figure 1. Relationship between log-transformed maternal blood cadmium (Cd) levels and Korean attention-deficit/hyperactivity disorder rating scale (K-ARS) total score in 6-yearold girls $(p<0.001)$. secondhand tobacco smoke exposure, K-ARS scores, and maternal blood heavy metal ( $\mathrm{Pb}$ and $\mathrm{Cd}$ ) levels are presented in Table 1. The mean maternal age of the 479 mothers was 31.4 years, and $84.3 \%$ of the mothers had an education level of university or above. Only $4.4 \%$ of the mothers were current smokers. The mean age of the children was 71.1 months, and $53.4 \%$ were male. Furthermore, $60.9 \%$ of the boys and $61.0 \%$ of the girls were the first-born child in the family. Regarding ADHD symptoms, the mean total K-ARS score was 6.9 for boys, which was higher than the mean total K-ARS score for girls (5.4). This relationship was also observed when symptoms were classified by type (attention-deficit or hyperactivity); the K-ARS scores in boys and girls for the attention-deficit type were 3.9 and 3.0, respectively, and those for the hyperactivity type were 3.0 and 2.3, respectively. The mean maternal blood levels of $\mathrm{Pb}$ and $\mathrm{Cd}$ were $1.4 \mu \mathrm{g} / \mathrm{dL}$ and $0.7 \mu \mathrm{g} / \mathrm{L}$, respectively. No statistically significant differences in any demographic or clinical factors were observed according to sex.

Figure 1 shows the association between prenatal blood $\mathrm{Cd}$ concentrations and the K-ARS total score. A positive, nearly linear association was observed between prenatal $C d$ levels and the K-ARS total score at 6 years of age. Therefore, assuming linearity, we used a generalized estimating equation model in our analysis. When we performed an additional analysis assuming a non-linear association, similar results to our main analysis were observed (Supplemental Material 1). Table 2 shows estimations of the relationships between prenatal blood $\mathrm{Cd}$ levels and the total and subcategory scores for ADHD as shown

Table 2. Percent change in K-ARS scores associated with 2-fold increases in the maternal blood cadmium level in 6-year old children

\begin{tabular}{llcr}
\hline Subtype & Sex & \% change $\mathbf{( 9 5 \% ~ C I )}$ & $\boldsymbol{p}$-value \\
\hline Total & Overall & $6.4(0.1,13.2)$ & 0.045 \\
& Male & $-0.1(-8.3,8.6)$ & 0.964 \\
\multirow{3}{*}{ Attention deficit } & Female & $22.3(11.6,34.1)$ & $<0.001$ \\
& Overall & $7.8(-0.6,17.0)$ & 0.070 \\
& Male & $1.8(-9.0,14.1)$ & 0.747 \\
Hyperactivity & Female & $21.2(7.4,36.8)$ & 0.002 \\
& Overall & $4.5(-4.7,14.8)$ & 0.347 \\
& Male & $-2.9(-14.6,10.4)$ & 0.653 \\
& Female & $23.5(7.1,42.3)$ & 0.004 \\
\hline
\end{tabular}

The model was adjusted for sex (male or female), parity, birth weight $(\mathrm{kg})$, mother's body mass index, mother's education level, mother's age, and mother's passive smoking status.

K-ARS, Korean attention-deficit/hyperactivity disorder rating scale; Cl, confidence interval. 
by percentage increase in the K-ARS score per 2-fold increase in the prenatal blood Cd level. No significant associations were observed between postnatal (concurrent) Cd exposure at 6 years and K-ARS scores. However, each doubling of the maternal prenatal Cd level was significantly associated with an increase in the K-ARS total score of $22.3 \%$ ( $95 \%$ confidence interval [CI], 11.6 to 34.1 ) in girls only. When investigated separately by symptom type, again in girls only, each doubling of prenatal $\mathrm{Cd}$ exposure showed a $21.2 \%(95 \% \mathrm{Cl}, 7.4$ to 36.8$)$ increase in the score related to attention-deficit symptoms and a $23.5 \%$ (95\% $\mathrm{Cl}, 7.1$ to 42.3 ) increase in the score for the hyperactivity symptoms. These associations were not observed for boys. The effect size was significantly larger in girls than in boys $(p=0.002)$. A sensitivity analysis with adjustment for maternal blood $\mathrm{Pb}$ levels and concurrent blood Cd levels at age 6 did not change the main findings. In our analysis, maternal $\mathrm{Pb}$ levels were not associated with K-ARS scores (Supplemental Material 2). When we included concurrent (6-year-old) blood Cd levels and maternal $\mathrm{Pb}$ levels in our model, the main results remained unchanged (Supplemental Material 2). The proportion of current smokers in our population was very low (4.4\%), and similar results were seen after we excluded current smokers (Supplemental Material 3).

\section{DISCUSSION}

In this study, we investigated the relationship between prenatal blood Cd exposure and ADHD symptoms in 6-year-old children. We observed that higher maternal blood Cd concentrations during pregnancy were significantly associated with higher ADHD scores, as measured by the K-ARS questionnaire, in girls.

To date, there is only limited information on the relationship between $\mathrm{Cd}$ exposure and ADHD. The published literature lacks significant findings and contains mixed results. A study conducted using National Health and Nutrition Examination Survey data from 1999-2000 reported non-significantly higher odds of ADHD diagnosis in children with higher blood Cd concentrations, but this trend disappeared when the analysis was adjusted for the effects of persistent organic pollutants [19]. Another study reported that a higher frequency of social problems, withdrawal, and attention problems was linked to higher concentrations of $C d$ in children's hair at ages 7 years to 16 years [27]. In contrast, another recent analysis of National Health and Nutrition Examination Survey data from 1997-2004 found a non-significant decrease in ADHD risk with increasing levels of urinary Cd in children [28].

Although an association between $\mathrm{Cd}$ and ADHD was not consistently found in previous studies, numerous studies have investigated the behavioral effects of early-life $\mathrm{Cd}$ exposure and have reported significant findings. In Belgium, a prospective study of children reported a significant association between prenatal exposure to $\mathrm{Cd}$ and an increased risk of emotional problems among 7- to 8-year-old boys [29]. Another study in China reported an association between attention and social problems in 7- to 16-year-old children and increased hair Cd levels [27].

Previous animal studies have shown that $\mathrm{Cd}$ affects central nervous system metabolism via inhibition of sulfhydryl-containing enzymes. As a result, in chronically exposed individuals, Cd can act as a depressant of various neurotransmitters, including acetylcholine, norepinephrine, and serotonin [30,31]. Changes in the metabolism of the dopamine and serotonin systems were also documented, and these neurotransmitter systems are known to be linked with the etiology of ADHD [32]. Animal studies have also shown that $\mathrm{Cd}$ can readily cross the blood-brain barrier [33], from which we can infer that Cd may move directly to the central nervous system and impact the neurodevelopment of young children [34]. Other studies have found evidence that $\mathrm{Cd}$ exposure can induce changes in activity levels in animals, although these studies showed inconsistent results, as some reported increased hyperactivity, while others found a decrease in exploratory activity and in time spent in motion [4].

We tested maternal blood $\mathrm{Pb}$ levels as a potential covariate in the relationship between blood Cd levels and K-ARS scores, and our analysis found that maternal blood $\mathrm{Pb}$ concentrations were not associated with K-ARS scores. Specific domains of neurobehavioral impairment that may be associated with $\mathrm{Pb}$ exposure remain an issue of uncertainty in the literature. Previous studies have displayed inconsistent results regarding the contribution of $\mathrm{Pb}$ exposure to inattention and impulsivity [35]. Maternal Cd levels, on the other hand, displayed a positive relationship with ADHD in girls when included in the model along with maternal $\mathrm{Pb}$ levels and concurrent blood $\mathrm{Cd}$ levels in the children.

The main strength of this study is its prospective cohort design. We also controlled for potential confounders, such as blood $\mathrm{Pb}$ levels and secondhand smoking status. However, this study also has some limitations. The current study investi- 
gated the associations between toxic heavy metal exposure and K-ARS scores, and although we adjusted for maternal variables such as maternal age and education, we were not able to account for the diverse social, cultural, and socioeconomic effects that are known to play a role in the development of ADHD. The K-ARS, which was the screening tool used for the study, is fundamentally a parent-administered tool used to detect symptoms of ADHD in children. As this test was completed by parents of the children in the study, observer bias may have influenced the results. Consequently, there is potential for misclassification resulting from parents over-reporting or under-reporting their children's symptoms. Given this limitation, we attempted to show the associations between increased maternal levels of $\mathrm{Cd}$ and symptom-related scores of ADHD at 6 years of age, although the clinical significance of our findings needs further investigation. Additionally, no children in this cohort were clinically diagnosed with ADHD. The K-ARS scale is known to have high reliability and validity for the screening and diagnosis of ADHD, but it is not a clinical diagnostic tool [36]. Since the diagnosis of ADHD requires a thorough evaluation of consistent symptoms for more than 6 months by a trained physician, the results of this study should be interpreted with caution.

Our study is the first to suggest a significant dose-dependent relationship between prenatal $\mathrm{Cd}$ concentrations and ADHD scores at the age of 6 years. The strengths of our study are its prospective cohort design, as well as its consideration of multiple covariates, including prenatal $\mathrm{Pb}$ concentrations and concurrent blood $\mathrm{Cd}$ concentrations in the children at age 6. However, given the nature of parent-reported K-ARS scores, the results of this study should be interpreted cautiously. Additional prospective epidemiological studies should be performed to further replicate and clarify the possible association observed in this study.

\section{SUPPLEMENTAL MATERIALS}

Supplemental materials are available at https://doi.org/10. 3961/jpmph.19.175.

\section{CONFLICT OF INTEREST}

The authors have no conflicts of interest associated with the material presented in this paper.

\section{FUNDING}

This research was supported by a grant from the Ministry of Food and Drug Safety in 2018 (18162MFDS121) and from the Center for Environmental Health through the Ministry of Environment.

\section{ACKNOWLEDGEMENTS}

None.

\section{AUTHOR CONTRIBUTIONS}

Conceptualization: WK, YCH. Data curation: YHL, BNK, CHS, YAL, JIK, YCH. Formal analysis: WK, YJ, YHL, YCH. Funding acquisition: YCH. Methodology: WK, YHL, YCH. Project administration: YCH. Visualization: YCH. Writing - original draft: WK. Writing - review \& editing: WK, YJ, YCH, YHL, BNK, CHS, YAL, JIK.

\section{ORCID}

Woosung Kim https://orcid.org/0000-0003-3404-5576

Yoonyoung Jang https://orcid.org/0000-0002-8161-9950

Youn-Hee Lim https://orcid.org/0000-0002-1290-5814

Bung-Nyun Kim https://orcid.org/0000-0002-2403-3291

Choong Ho Shin https://orcid.org/0000-0002-9813-1134

Young Ah Lee https://orcid.org/0000-0001-9179-1247

Johanna Inhyang Kim https://orcid.org/0000-0002-2367-

0934

Yun-Chul Hong https://orcid.org/0000-0001-9010-7271

\section{REFERENCES}

1. Agency for Toxic Substances and Disease Registry. Toxicological profile for cadmium; 2012 [cited 2019 Jun 1]. Available from: https://www.atsdr.cdc.gov/ToxProfiles/tp5.pdf.

2. Järup L, Akesson A. Current status of cadmium as an environmental health problem. Toxicol Appl Pharmacol 2009;238(3): 201-208.

3. European Food Safety Authority (EFSA). Cadmium in food-scientific opinion of the Panel on Contaminants in the Food Chain. EFSA J 2009;7(3):980.

4. Ali MM, Murthy RC, Chandra SV. Developmental and longterm neurobehavioral toxicity of low level in-utero cadmium exposure in rats. Neurobehav Toxicol Teratol 1986;8(5):463-468. 
5. Dési I, Nagymajtényi L, Schulz H. Behavioural and neurotoxicological changes caused by cadmium treatment of rats during development. J Appl Toxicol 1998;18(1):63-70.

6. Lehotzky K, Ungváry G, Polinák D, Kiss A. Behavioral deficits due to prenatal exposure to cadmium chloride in CFY rat pups. Neurotoxicol Teratol 1990;12(2):169-172.

7. Nagymajtényi L, Schulz H, Dési I. Behavioural and functional neurotoxicological changes caused by cadmium in a threegenerational study in rats. Hum Exp Toxicol 1997;16(12):691699.

8. Nation JR, Bourgeois AE, Clark DE, Baker DM, Hare MF. The effects of oral cadmium exposure on passive avoidance performance in the adult rat. Toxicol Lett 1984;20(1):41-47.

9. Jiang HM, Han GA, He ZL. Clinical significance of hair cadmium content in the diagnosis of mental retardation of children. Chin Med J (Engl) 1990;103(4):331-334.

10. Marlowe M, Errera J, Jacobs J. Increased lead and cadmium burdens among mentally retarded children and children with borderline intelligence. Am J Ment Defic 1983;87(5):477-483.

11. Thatcher RW, Lester ML, McAlaster R, Horst R. Effects of low levels of cadmium and lead on cognitive functioning in children. Arch Environ Health 1982;37(3):159-166.

12. Capel ID, Pinnock MH, Dorrell HM, Williams DC, Grant EC. Comparison of concentrations of some trace, bulk, and toxic metals in the hair of normal and dyslexic children. Clin Chem 1981; 27(6):879-881.

13. Ely DL, Mostardi RA, Woebkenberg N, Worstell D. Aerometric and hair trace metal content in learning-disabled children. Environ Res 1981;25(2):325-339.

14. Pihl RO, Parkes M. Hair element content in learning disabled children. Science 1977;198(4313):204-206.

15. Thatcher RW, McAlaster R, Lester ML, Cantor DS. Comparisons among $E E G$, hair minerals and diet predictions of reading performance in children. Ann NY Acad Sci 1984;433:87-96.

16. Marlowe M, Cossairt A, Moon C, Errera J, MacNeel A, Peak R, et al. Main and interaction effects of metallic toxins on classroom behavior. J Abnorm Child Psychol 1985;13(2):185-198.

17. Cao Y, Chen A, Radcliffe J, Dietrich KN, Jones RL, Caldwell K, et al. Postnatal cadmium exposure, neurodevelopment, and blood pressure in children at 2, 5, and 7 years of age. Environ Health Perspect 2009;117(10):1580-1586.

18. Gillberg C, Norén JG, Wahlström J, Rasmussen P. Heavy metals and neuropsychiatric disorders in six-year-old children. Aspects of dental lead and cadmium. Acta Paedopsychiatr 1982;48(5): 253-263.
19. Lee DH, Jacobs DR, Porta M. Association of serum concentrations of persistent organic pollutants with the prevalence of learning disability and attention deficit disorder. J Epidemiol Community Health 2007;61(7):591-596.

20. Willcutt EG. The prevalence of DSM-IV attention-deficit/hyperactivity disorder: a meta-analytic review. Neurotherapeutics 2012;9(3):490-499.

21. Faraone SV, Biederman J, Mick E. The age-dependent decline of attention deficit hyperactivity disorder: a meta-analysis of follow-up studies. Psychol Med 2006;36(2):159-165.

22. Fayyad J, Sampson NA, Hwang I, Adamowski T, Aguilar-Gaxiola S, Al-Hamzawi A, et al. The descriptive epidemiology of DSM-IV adult ADHD in the World Health Organization World Mental Health Surveys. Atten Defic Hyperact Disord 2017;9(1):47-65.

23. Faraone SV, Biederman J, Mick E. The age-dependent decline of attention deficit hyperactivity disorder: a meta-analysis of follow-up studies. Psychol Med 2006;36(2):159-165.

24. Biederman J, Petty CR, Clarke A, Lomedico A, Faraone SV. Predictors of persistent ADHD: an 11-year follow-up study. J Psychiatr Res 2011;45(2):150-155.

25. Biederman J, Petty CR, O'Connor KB, Hyder LL, Faraone SV. Predictors of persistence in girls with attention deficit hyperactivity disorder: results from an 11-year controlled follow-up study. Acta Psychiatr Scand 2012;125(2):147-156.

26. DuPaul GJ, Reid R, Anastopoulos AD, Lambert MC, Watkins MW, Power TJ. Parent and teacher ratings of attention-deficit/hyperactivity disorder symptoms: factor structure and normative data. Psychol Assess 2016;28(2):214-225.

27. Bao QS, Lu CY, Song H, Wang M, Ling W, Chen WQ, et al. Behavioural development of school-aged children who live around a multi-metal sulphide mine in Guangdong province, China: a cross-sectional study. BMC Public Health 2009;9:217.

28. Ciesielski T, Weuve J, Bellinger DC, Schwartz J, Lanphear B, Wright RO. Cadmium exposure and neurodevelopmental outcomes in U.S. children. Environ Health Perspect 2012;120(5): 758-763.

29. Sioen I, Den Hond E, Nelen V, Van de Mieroop E, Croes K, Van Larebeke $\mathrm{N}$, et al. Prenatal exposure to environmental contaminants and behavioural problems at age 7-8 years. Environ Int 2013;59:225-231.

30. Singhal RL, Merali Z, Hrdina PD. Aspects of the biochemical toxicology of cadmium. Fed Proc 1976;35(1):75-80.

31. Stowe HD, Wilson M, Goyer RA. Clinical and morphologic effects of oral cadmium toxicity in rabbits. Arch Pathol 1972; 94(5):389-405. 
32. Faraone SV, Perlis RH, Doyle AE, Smoller JW, Goralnick JJ, Holmgren MA, et al. Molecular genetics of attention-deficit/hyperactivity disorder. Biol Psychiatry 2005;57(11):1313-1323.

33. Andersson $\mathrm{H}$, Petersson-Grawé $\mathrm{K}$, Lindqvist $\mathrm{E}$, Luthman J, Oskarsson A, Olson L. Low-level cadmium exposure of lactating rats causes alterations in brain serotonin levels in the offspring. Neurotoxicol Teratol 1997;19(2):105-115.

34. Provias JP, Ackerley CA, Smith C, Becker LE. Cadmium encephalopathy: a report with elemental analysis and pathological findings. Acta Neuropathol 1994;88(6):583-586.

35. Goodlad JK, Marcus DK, Fulton JJ. Lead and attention-deficit/ hyperactivity disorder (ADHD) symptoms: a meta-analysis. Clin Psychol Rev 2013;33(3):417-425.

36. Park Jl, Shim SH, Lee M, Jung YE, Park TW, Park SH, et al. The validities and efficiencies of Korean ADHD rating scale and Korean child behavior checklist for screening children with ADHD in the community. Psychiatry Investig 2014;11(3):258265. 